\title{
Hábitos de estudos de graduandos em Ciência da Computação e Engenharia de Software: uma análise no período de pandemia
}

\author{
Flávia A. Barbosa, Ícaro M. Crespo, Lucas A. G. Garais, Andréa S. Bordin \\ ${ }^{1}$ Universidade Federal do Pampa (Unipampa) - Campus Alegrete \\ Av. Tiarajú, 810 - 97.546-550 - Alegrete - RS - Brazil \\ \{flaviabarbosa.aluno, icarocrespo.aluno\}@unipampa.edu.br \\ \{lucasgarais.aluno, andreabordin\}@unipampa.edu.br
}

\begin{abstract}
During the pandemic period, the teaching activities from most universities were suspended. As a consequence, it was observed that a lot of students felt unmotivaded. This study objective to analise study habits of Computer Science (CS) and Software Engineering (SE) students from Unipampa/Alegrete. For this, a survey was carried out in which 81 responses were obtained. The data analisys revealed that students of SE felt more motivated and dedicatd more time to studies, unlike the CS students who indicated they miss the face-to-face classes. The results of this research can be used to plan remote teaching activities, as well as assist in the curriculum management of the courses in question.
\end{abstract}

Resumo. Durante o período de pandemia, as atividades em grande parte das universidades foram suspensas. Como consequência, observou-se que muitos estudantes se sentiram desmotivados. Este estudo objetiva analisar os hábitos de estudos de alunos dos cursos de Ciência da Computação (CC) e Engenharia de Software (ES) da Unipampa/Alegrete. Para isso, foi realizado um survey no qual foram obtidas 81 respostas. A análise dos dados revelou que alunos de ES se sentiram mais motivados e dedicaram mais tempo aos estudos, diferente dos alunos de CC que indicaram sentir falta das aulas presenciais. Os resultados desta pesquisa podem ser utilizados para o planejamento de atividades de ensino remotas, assim como auxiliar na gestão curricular dos cursos em questão.

\section{Introdução}

O ano de 2020 trouxe uma mudança brusca em diversos segmentos da sociedade em virtude da pandemia do vírus Sars-CoV-2 (Síndrome Respiratória Aguda Grave) [BRASIL 2020b]. Como forma de prevenção à pandemia foram aplicadas medidas de isolamento e distanciamento social. As aulas presenciais nos diferentes níveis educacionais foram suspensas, por decisão do Ministério da Educação brasileiro [BRASIL 2020a].

De acordo com [Van Nguyen et al. 2020], a parada repentina neste aprendizado resulta em perda de interesse em adquirir novos conhecimentos. Como consequências desta ação, podemos citar queda no rendimento, alienação, desmotivação para estudar e baixa na participação em atividades acadêmicas, além de perda do sentimento de pertencimento aos estabelecimentos de ensino [Marques 2020]. 
Como alternativas à suspensão de atividades presenciais, muitas instituições de ensino propuseram ações online nos eixos de ensino, pesquisa e extensão para a manutenção do vínculo proporcionado pelo ensino da universidade. Na Universidade Federal do Pampa (Unipampa), campus Alegrete, ações com este objetivo também foram desenvolvidas. Dentre essas ações, destaca-se o I Ciclo Online de Palestras dos cursos de Ciência da Computação (CC) e Engenharia de Software (ES).

O presente estudo tem como motivação a avaliação das palestras do ciclo supracitado, onde questionou-se sobre hábitos de estudos no período de isolamento. A análise das respostas revelou que parte dos alunos dedicava pouco tempo às atividades de estudo, assim como apresentava um quadro de desânimo à situação de isolamento. No entanto, outra parte manifestou que estava buscando estudar assuntos da área de Computação. Como a avaliação era voluntária, o número de respondentes não foi expressivo. Dessa forma, entendeu-se que era necessário estender esses questionamentos para um maior número de discentes de $\mathrm{CC}$ e ES.

Uma busca na literatura encontrou estudos que investigaram os diversos impactos da pandemia de COVID-19 no ensino de uma forma mais global. O estudo de [Van Nguyen et al. 2020] apresenta dados sobre o comportamento e percepção de estudantes de graduação de universidades do Vietnã, onde cerca de $81 \%$ dos respondentes alegaram que a COVID-19 afetou significativamente as atividades de trabalho/estudo. Além desse, o estudo de [Onyema et al. 2020] analisou os impactos da pandemia em diferentes graus da educação de diferentes países, no qual mais de $70 \%$ dos participantes concordaram que infraestrutura inadequada, como falta de computadores e internet, foi o principal fator que limitou seu envolvimento na educação online. Não foram encontrados estudos especificamente relacionados a alunos de graduação da área de Computação.

Neste artigo é apresentada uma análise dos hábitos de estudos e envolvimento em atividades acadêmicas dos alunos dos cursos de Ciência da Computação e Engenharia de Software da UNIPAMPA durante o período de isolamento da pandemia. Para isso, foi conduzido um survey com questões demográficas, de infraestrutura e de hábitos de estudos. Dentre as contribuições deste estudo estão a identificação de perfis de alunos com hábitos de estudos diferentes durante a pandemia, assim como a compilação das fontes de estudos mais utilizadas pelos alunos e as áreas e assuntos pelos quais mais se interessaram.

\section{Metodologia}

O survey desta pesquisa seguiu o processo proposto por [Kasunic 2005], no qual tem-se as seguintes etapas: Planejamento, Elaboração, Teste piloto, Execução e Análise. Na etapa de planejamento, o escopo e o conjunto de questões da pesquisa aperfeiçoaram-se por meio de reuniões entre os pesquisadores. Definiu-se uma pesquisa de caráter quantitativo e qualitativo, direcionada a discentes dos cursos de CC e ES da Unipampa. Embasado nos tipos de amostragem da pesquisa, optou-se pela técnica probabilística de amostragem aleatória simples, na qual todos os elementos da população, ou seja, alunos dos cursos da área de Computação, têm a mesma probabilidade de pertencerem à amostra [Grzybovski 2005].

Na etapa de elaboração, definiu-se o mecanismo utilizado para a coleta de dados, 
sendo este um formulário disponibilizado via plataforma Google Forms ${ }^{1}$. O formulário constituiu-se em 18 questões subdivididas em 5 grupos, sendo eles: identificação; infraestrutura necessária ao estudo/pesquisa; hábitos de estudos; fontes de consultas e atividades acadêmicas.

Para efeito de teste e refatoração de possíveis inconsistências no instrumento, na etapa de teste piloto o instrumento foi aplicado inicialmente a um grupo focal, constituído por 2 discentes de ES e 1 discente de CC. Os discentes foram encorajados a apontar problemas pertinentes ao instrumento de pesquisa. Feito isso, cumpriu-se a análise e correção dos potenciais problemas. Um novo pré-teste foi realizado, desta vez utilizando 8 discentes, sendo 2 de CC e 6 de ES. A versão final das questões é exibida na Tabela 1. O convite de participação na pesquisa foi feito para todos os alunos dos dois cursos (348 matriculados), por meio do endereço eletrônico institucional. Além desse meio, redes sociais foram utilizadas para maior alcance de participações.

Na etapa de análise de dados, primeiramente verificou-se a consistência e integridade das respostas obtidas na coleta. Ademais, realizou-se um processo de reestruturação nos dados para facilitar a análise. A reestruturação concretizou-se a partir da organização dos dados de forma ordenada em planilhas eletrônicas compartilhadas entre a equipe, de acordo com as questões e problemáticas impostas.

Tabela 1. Pesquisa sobre hábitos de estudos e envolvimento em atividades acadêmicas durante a pandemia

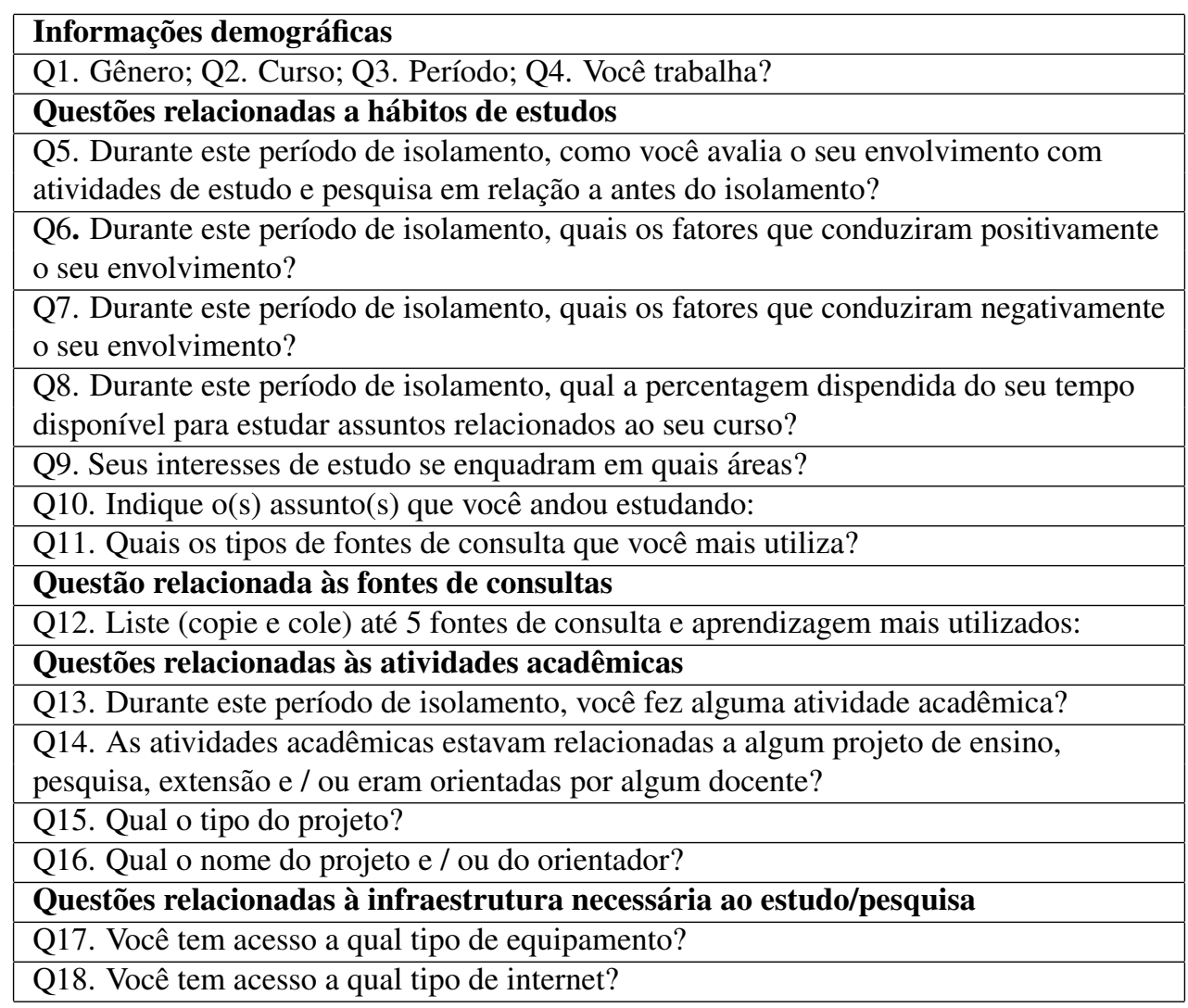

${ }^{1} \mathrm{O}$ instrumento de pesquisa pode ser acessado em: http://bit. ly/habitos Instrumento 


\section{Resultados}

Nesta seção, são apresentados os resultados obtidos a partir de 81 respostas, sendo 31 de discentes de CC e 50 de ES, representando, respectivamente, $17,7 \%$ e $29,0 \%$, dos alunos matriculados até o período deste estudo. Os dados são apresentados por grupos de questões, tal como no instrumento de coleta.

Em relação ao perfil demográfico dos respondentes, em ambos os cursos o percentual de participantes do sexo masculino foi bastante superior ao do sexo feminino. Destaca-se também, que o maior número de respondentes está cursando entre o $1^{\circ}$ e o $4^{\circ}$ semestre em ambos os cursos, e que o menor índice de participação no survey está entre os discentes do $5^{\circ}$ e $6^{\circ}$ semestre. Quando perguntados se trabalham, no curso de CC, $64,5 \%$ responderam que sim e 35,5\% que não. Já entre os respondentes de ES, $58 \%$ dos respondentes trabalham e $42,2 \%$ não.

$\mathrm{Na}$ análise referente às questões de infraestrutura necessária ao estudo/pesquisa, verificou-se que todos os respondentes possuem acesso a um ou mais aparelhos eletrônicos, sendo eles: celular, desktop/notebook e tablet. É válido ressaltar que em média 10,4\% (12,9\% de CC e 8,0\% de ES) dos respondentes afirmaram usar tablet, quando comparado ao uso de celular ou desktop/notebook. Entretanto, quando verifica-se qual tipo de internet os respondentes utilizam, os dados mostram que em média apenas 41,3\% (38,7\% de CC e 44,0\% de ES) dos discentes de ambos os cursos têm acesso a internet móvel. Em comparação, a banda larga mostrou ser utilizada por cerca de $99 \%$ dos respondentes, nota-se que o uso é consideravelmente maior.

Quanto aos hábitos de estudos durante a pandemia, foi possível perceber que 48,4\% dos respondentes de CC tiveram o seu envolvimento com atividades de estudo e pesquisa diminuídos. Em contrapartida, 40,0\% dos respondentes de ES apresentaram um aumento do envolvimento com atividades de estudo durante a pandemia, como visto na Figura1.

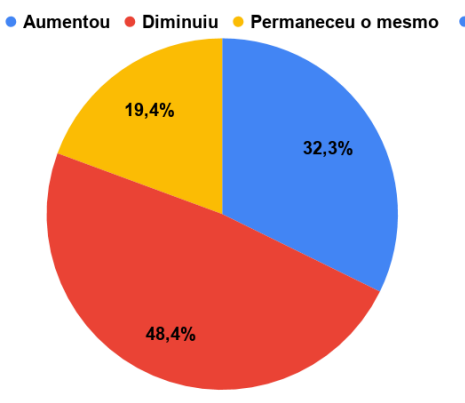

((a)) CC

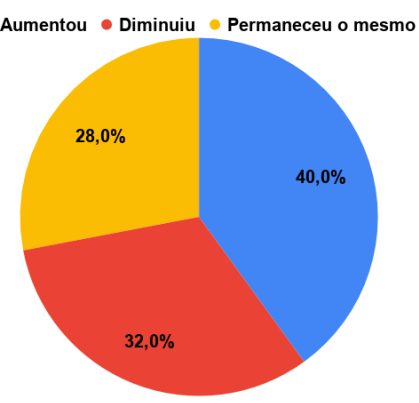

((b)) ES

Figura 1. Nível de envolvimento em atividades

A respeito de influências positivas nos hábitos de estudos, destacou-se o fator flexibilidade de tempo entre os discentes de ES e a liberdade de escolha dos assuntos estudados, entre os de CC. A frequência dos fatores infraestrutura da casa e proximidade com a família se sobressaíram na CC em relação à ES, como observado na Figura 2. Dentre as influências negativas, observa-se que três fatores foram mencionados por cerca de $30 \%$ a $40 \%$ dos discentes de ambos os cursos, são eles: problemas pessoais; falta de 
contato com colegas e professores; e preocupação com o cenário imposto pela pandemia. Em especial, o fator falta de aulas presenciais é salientado com $61,3 \%$ de frequência na $\mathrm{CC}$, como demonstrado na Figura 3

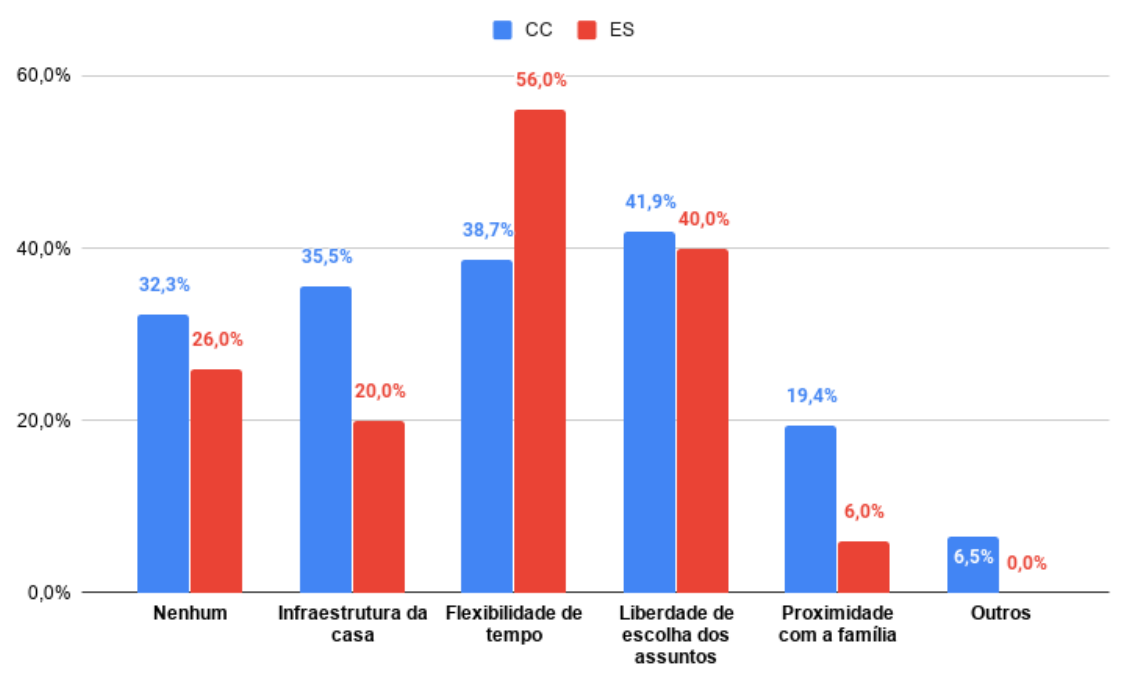

Figura 2. Influências positivas

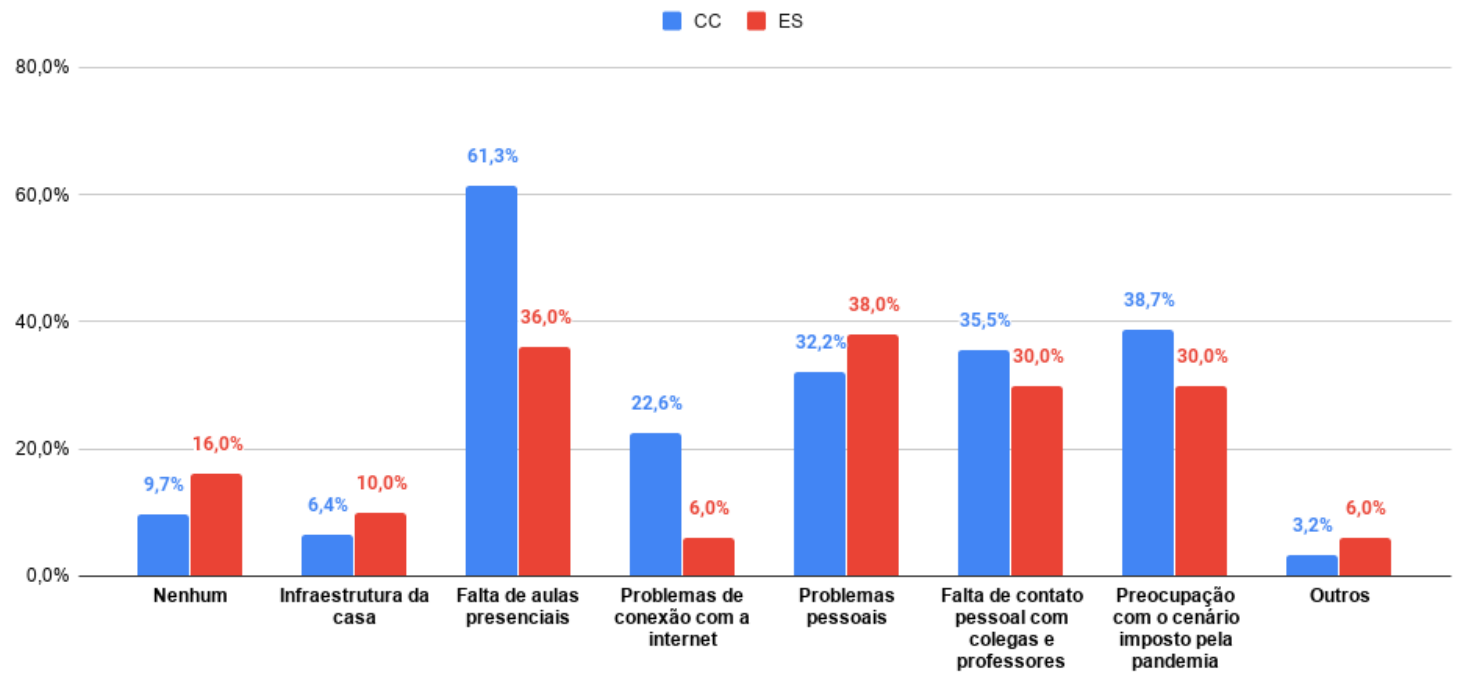

Figura 3. Influências negativas

Quando se refere ao tempo disponível dedicado a estudar, cerca de 38,7\% dos respondentes de CC destinaram entre 0 a 10,0\% do seu tempo para estudar assuntos relacionados ao seu curso. Por outro lado, houve uma polarização no que diz respeito aos respondentes de ES, com cerca de 20,0\% destinando pouco tempo (entre 0 a 10,0\%) e também aproximadamente 18,0\% dedicando mais da metade do tempo. Apenas 6,4\% dos participantes de CC dedicaram mais da metade do tempo para estudar, um contraste significativo em relação aos $18,0 \%$ de respondentes da ES que afirmaram o mesmo, como evidenciado na Figura 4. 


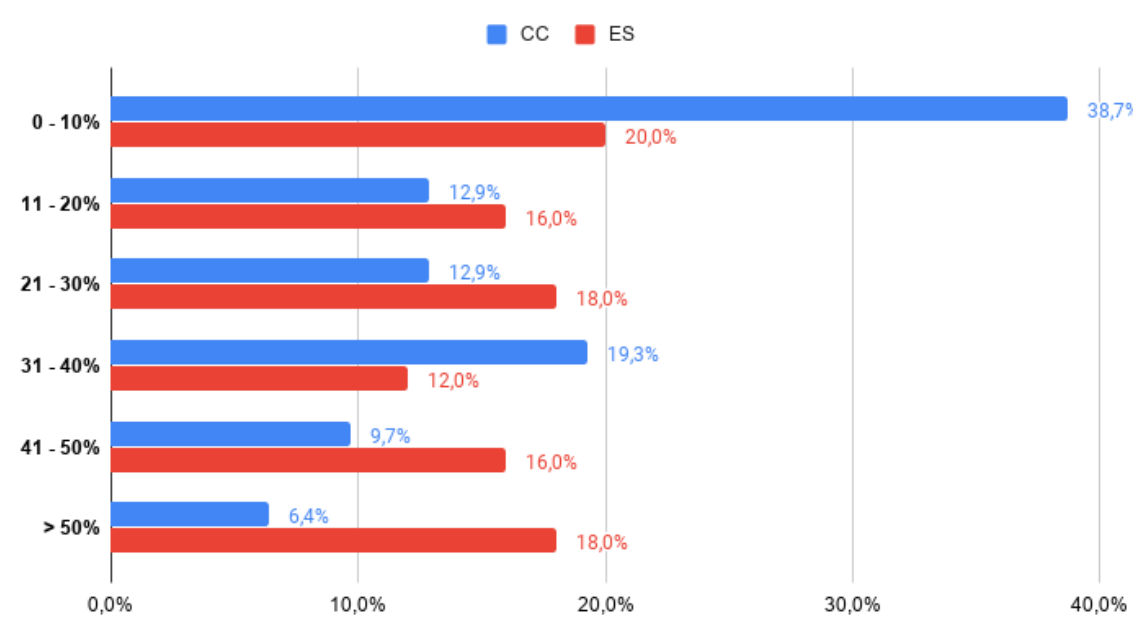

Figura 4. Percentagem de tempo disponível

Quanto às áreas de interesse de estudo, observa-se que o instrumento disponibilizava algumas áreas de conhecimento para alunos de CC e outras para alunos de ES, além de permitir a inserção de outras. Dentre aquelas consideradas pelos discentes da CC, Redes e Comunicações se sobressaiu em relação as demais com 35,5\% de frequência, sendo acompanhada por Banco de Dados (22,6\%) e Engenharia de Software (19,4\%). Outras áreas foram apontadas como de interesse pelos de CC, como: Desenvolvimento Web, Inglês, Data Science e Lógica de Programação, além disso, cerca de 22,6\% dos alunos afirmaram não ter interesse em estudar. No que diz respeito a assuntos estudados, dentre as respostas da CC, se destacam os tópicos: Redes e Telecomunicações, Javascript, Algoritmos e Programação, Python, Linguagem C e Cálculos Matemáticos. Como observado na Figura 5, outros temas relacionados à CC foram identificados, por exemplo: diversas linguagens de programação e tópicos matemáticos.

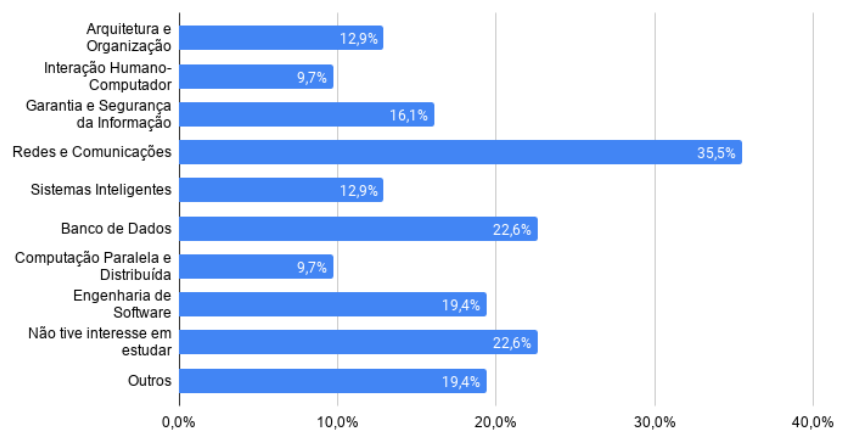

((a)) Áreas de Interesse

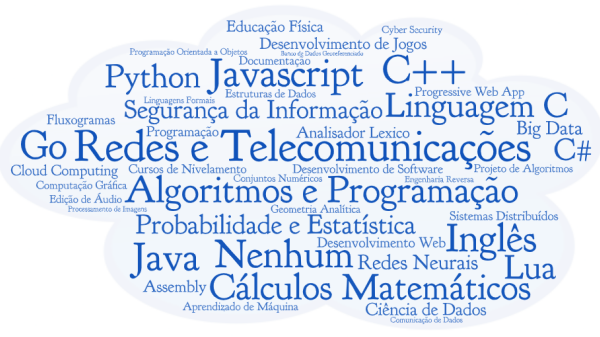

((b)) Assuntos

Figura 5. Áreas de interesse e assuntos da CC

Em relação às áreas de estudo escolhidas pelos participantes de ES durante o período de isolamento, Desenvolvimento de Software se sobreleva em relação as demais com 78,0\%, seguido das áreas: Arquitetura de Software $(38,0 \%)$ e Processos de Software $(30,0 \%)$. Ademais, $18,0 \%$ declararam que não tiveram interesse em estudar. No que se refere aos tópicos estudados pelos respondentes de ES, destacam-se os temas: Javascript, 
Desenvolvimento de Software, Mobile e Web, Arquitetura de Software, Python, Padrões de Projeto, dentre outros, como visto na Figura 6.

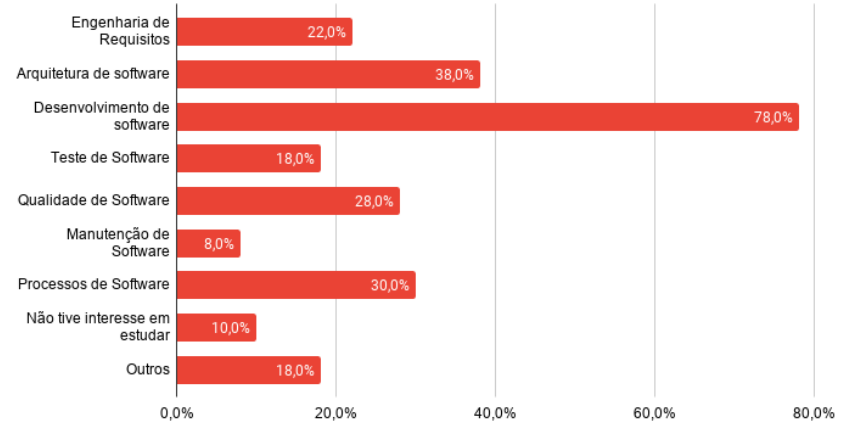

((a)) Áreas de Interesse

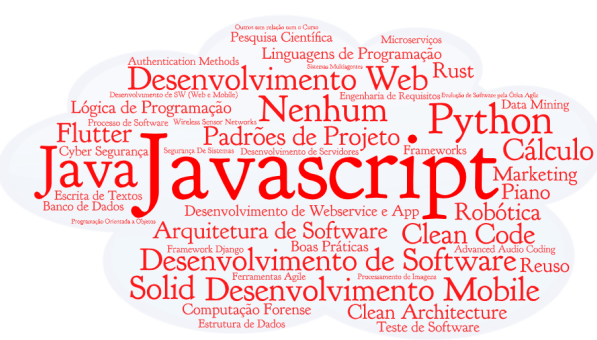

((b)) Assuntos

Figura 6. Áreas de interesse e assuntos da ES

Ainda em relação aos hábitos de estudos, foram analisadas as fontes de consulta ${ }^{2}$ mais utilizadas pelos discentes. Ao total foram informadas 203 fontes de consulta, sendo $83(48,9 \%)$ provenientes de alunos de CC e $120(59,1 \%)$ de ES. Para fins de categorização e análise dos dados, as fontes foram agrupadas e rotuladas em: AVA (Ambiente Virtual de Aprendizagem); blog; buscador; fórum; livro; página web de documentação; plataforma de cursos online; plataforma de versionamento; rede social; repositório científico; Youtube; e outros (para quando é impossível associar um link ao texto informado pelo respondente).

A Figura 7 mostra o percentual de fontes de consulta utilizadas pelos respondentes de CC e ES em cada grupo. Percebe-se que o Youtube é o mais utilizado pela CC $(29,1 \%)$ e aparece em segundo lugar para a ES com 20,0\%. Já as plataformas de cursos online são mais utilizadas pela ES $(25,8 \%)$. Na sequência, página web de documentação e blog, com o mesmo percentual, são mais utilizados pela CC. Quanto às fontes menos consultadas, tem-se rede social em ambos os cursos.

A análise das questões relacionadas às atividades acadêmicas ${ }^{3}$ revelou que 45,2\% dos respondentes de CC realizaram atividades acadêmicas durante a pandemia, contra $54,8 \%$ que não realizaram. Dentre os que realizaram atividades, $85,7 \%$ responderam que elas estavam relacionadas a algum projeto e/ou eram orientadas por algum docente $\mathrm{e}$ $14,3 \%$ responderam que não. Desses, $35,7 \%$ envolveram-se em projetos de ensino, $57,1 \%$ em projeto de pesquisa e $7,1 \%$ em projetos de extensão.

Já entre os respondentes de ES, 60,0\% realizaram atividades acadêmicas e 40,0\% não. Dentre os que realizaram atividades, $76,7 \%$ responderam que elas estavam relacionadas a algum tipo de projeto dentro da instituição e $23,3 \%$ responderam que não. Desses, $26,7 \%$ se envolveram em projetos de ensino, $60,0 \%$ de pesquisa e $13,3 \%$ de extensão.

\footnotetext{
${ }^{2}$ As fontes de consulta e os respectivos links podem ser acessadas em: https : / / bit. . 1y/32dh2 ki.

${ }^{3}$ São consideradas atividades acadêmicas quaisquer atividades de cunho organizacional ou de geração de conhecimento, relacionada à universidade. Exemplos: organização de eventos, atividades de iniciação científica (pesquisa), dentre outros.
} 


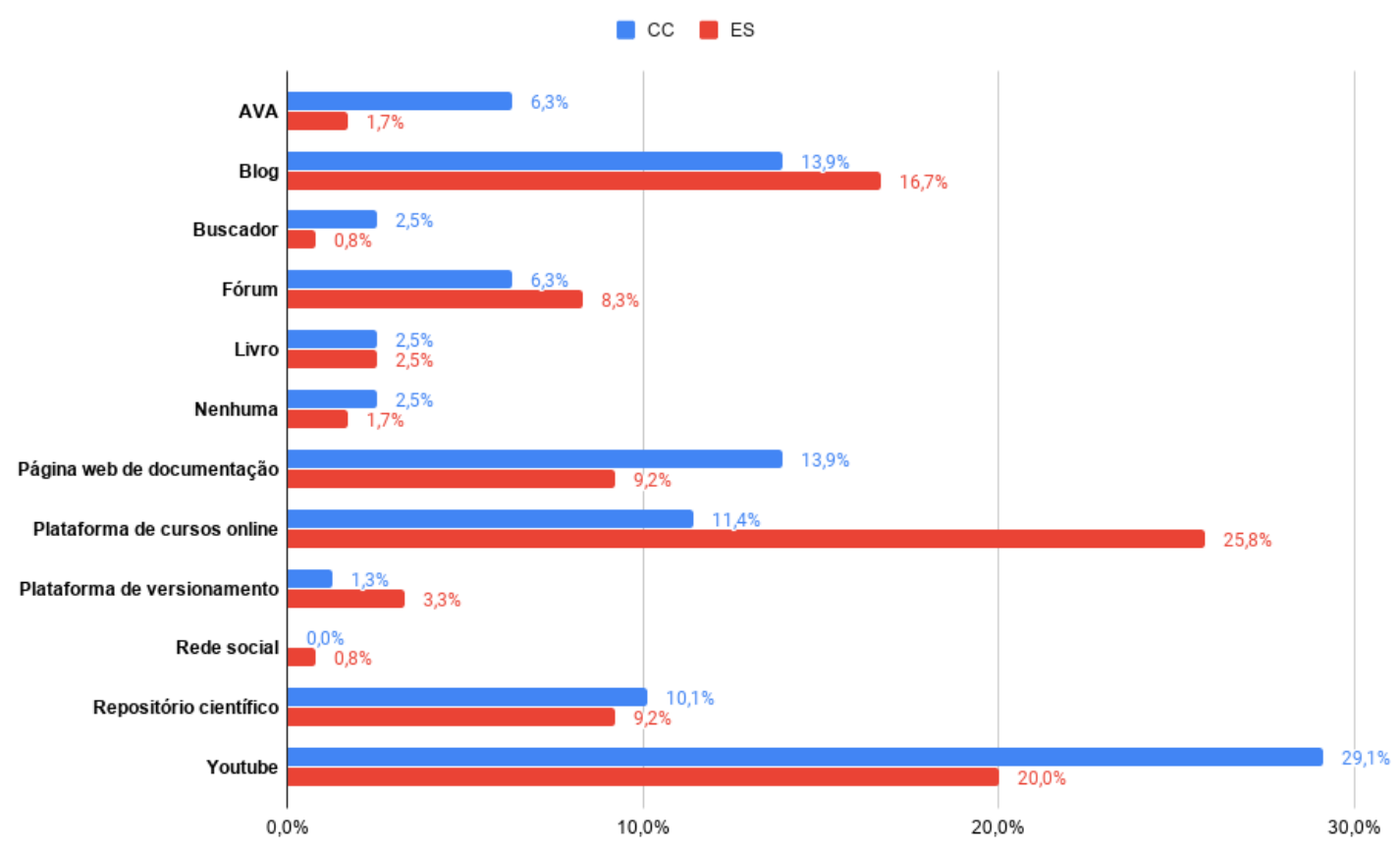

Figura 7. Fontes compiladas

\section{Discussão}

Em relação à infraestrutura, entende-se que o cenário de acessibilidade positivo de ambos os cursos esteja relacionado ao fato de que a grande maioria dos alunos de Computação já possui acesso a essas ferramentas, uma vez que são necessárias para a realização das atividades dos cursos. No entanto, existe a possibilidade que não reflita a realidade da maioria, uma vez que a pesquisa não atingiu a totalidade de matriculados.

A diminuição do nível de envolvimento em atividades de estudo entre os alunos de $\mathrm{CC}$, pode estar relacionado com a falta de aulas presenciais, apontado como influência negativa pelos próprios. Além disso, foram os que dispenderam menos tempo aos estudos e os que menos realizaram atividades acadêmicas. Já entre os alunos de ES, o nível de envolvimento aumentou, o que, como mencionado por eles, pode ter sido influenciado pela flexibilidade de tempo. Ademais, foram os que mais dispenderam tempo aos estudos e os que realizaram mais atividades acadêmicas.

Esse cenário caracteriza alunos com perfis diferentes e pode estar relacionado à metodologia de ensino empregada nos diferentes cursos. O curso de CC é conduzido através de metodologia de ensino tradicional, onde os componentes curriculares são ministrados de uma forma essencialmente expositiva. Já no curso de ES é adotada a metodologia ativa de Aprendizagem Baseada em Problemas (ABP) com uma carga horária significativa, na qual os alunos são desafiados a resolver problemas, sendo estimulados a aprender de forma autodidata. Dessa forma, alunos de CC podem se sentir menos motivados a estudar de forma autônoma, sentindo falta das aulas presenciais, ao contrário dos alunos de ES, que, em função da ABP, se sentem mais confortáveis em fazê-lo.

Dentre os fatores, elencados por ambos os cursos, que mais influenciaram positivamente o envolvimento em atividades, aparece a flexibilidade de tempo. Entende-se que 
as demandas dos cursos em tempos normais exigem uma gestão de tempo mais rigorosa por parte dos alunos, o que não foi necessário durante a pandemia, onde eles possivelmente conseguiram gerenciar melhor o seu tempo, sendo capazes de estipular uma rotina de estudos adaptada a diferentes horários e condições. Na sequência tem-se liberdade de escolha dos assuntos, que pode estar relacionada à diversidade e flexibilidade que a área de Computação oferece, o que por sua vez incentiva os estudantes a serem mais curiosos e buscarem novos conhecimentos.

Ainda em relação às influências positivas, a infraestrutura da casa e a proximidade com a família foram mais apontados pelos discentes de CC, com um significativo percentual de diferença em relação aos de ES. Entende-se que isso possa estar relacionado a um perfil de aluno que, em sala de aula, é exposto a um ambiente de aprendizagem tradicional, mais estável e controlado. Nessa linha, os discentes da CC tendem a encontrar tais características nos fatores supracitados.

Sobre as três influências negativas que impactaram o envolvimento com os estudos, apontadas de forma equilibrada por ambos os cursos, entende-se que são fatores esperados diante deste cenário de isolamento devido à pandemia, independentemente de área de conhecimento. A população em geral vem apresentando problemas de natureza pessoal, como por exemplo, problemas de convivência com a família, problemas financeiros, problemas psicológicos, dentre outros. A ausência de contato pessoal tende a prejudicar o envolvimento com os estudos. Segundo [Riess 2010] a sociabilização é um fator importante para a aprendizagem, uma vez que ela possibilita a troca de conhecimentos e a sedimentação dessa aprendizagem.

Em relação a assuntos estudados pelos respondentes de CC, predominam aqueles associados a linguagens de programação científicas, como C++, C, Lua, Python. Tal ocorrência parece estar alinhada ao perfil de alunos deste curso, que oferta em sua estrutura curricular componentes curriculares que demandam a utilização de tais linguagens. Por outro lado, na ES a área de desenvolvimento de software e os assuntos relacionados a essa área, como as linguagens para desenvolvimento de aplicações web e apps, teve um expressivo destaque. Isso também parece estar alinhado ao perfil de alunos de ES, onde a metodologia ABP, adotada nas disciplinas de Resolução de Problemas, demanda aos discentes o desenvolvimento de aplicações utilizando diferentes tecnologias.

Uma grande adesão a meios eletrônicos para consulta foi vista nos dados. A evidente preferência pelo Youtube e pelas plataformas de cursos online pode ser reflexo de que ambos os tipos de fonte oferecem conteúdo de uma forma semelhante à sala de aula, com uma pessoa (ou uma voz) falando enquanto apresenta algo, o que provavelmente faz com que os alunos se sintam mais familiarizados com esses meios. Mais especificamente, em relação à preferência de ES pelas plataformas de cursos online, entende-se que, novamente, possa estar relacionado ao perfil autodidata que os alunos apresentam em função da metodologia utilizada no curso. Da mesma forma, entende-se que a escolha de CC por páginas web de documentação, parece estar alinhado a um perfil de discente mais acostumado a acessar uma informação no seu estado original, sem subterfúgios.

\section{Considerações Finais}

As evidências indicam que alunos de Ciência da Computação e Engenharia de Software, apesar de serem da área de Computação, se comportaram de forma diferente durante 
a pandemia, no que diz respeito aos hábitos de estudos e envolvimento em atividades acadêmicas. Alunos de ES tiveram maior participação na pesquisa e demostraram ter uma postura mais ativa, o que parece estar relacionado à metodologia $\mathrm{ABP}$.

Ademais, o entendimento dos perfis permite que sejam tomadas decisões em nível de gestão curricular dos cursos, independentemente de período de isolamento. O mapeamento das áreas e assuntos de interesse podem auxiliar na decisão de oferta de componentes curriculares complementares ou eletivos e podem gerar pontos de reflexão em discussões sobre estruturação curricular, dentre outros.

Como ameaças à validade da pesquisa destaca-se o número da amostra que, para efeitos de generalização dos resultados, ficou aquém do esperado. É válido ressaltar que os resultados refletem majoritariamente os dados de alunos do gênero masculino, matriculados nos dois primeiros anos dos cursos.

Como trabalhos futuros, pretende-se aprimorar o survey para contemplar questões relacionadas à hábitos de estudos em contextos tanto de aulas remotas como de aulas presenciais, permitindo também a análise do impacto das metodologias de ensino distintas aplicadas nos dois cursos. Ademais, deseja-se investigar o processo de escolha das fontes de consultas por partes dos alunos de CC e ES e, a partir disso, propor um processo automatizado de curadoria colaborativo, onde alunos e professores possam interagir para qualificar as fontes de consulta mais relevantes.

\section{Referências}

BRASIL, M. d. E. (2020a). Portaria $\mathrm{n}^{0}$ 343, de 17 de março de 2020. Disponível em: https://pesquisa.in.gov.br/imprensa/jsp/visualiza/index.jsp?data=18/03/2020jornal=515 \&pagina $=39$. Acessado em 11 de ago. de 2020.

BRASIL, M. d. S. (2020b). Sobre a doença. Disponível em: https://coronavirus.saude.gov.br/sobre-a-doenca. Acessado em 13 de ago. de 2020.

Grzybovski, D. (2005). Revisão teórica sobre pesquisa quantitativa, mensuração, amostragem e análise multivariada. Texto para Discussão, (13):2005.

Kasunic, M. (2005). Designing an effective survey. Technical report, Carnegie-Mellon Univ Pittsburgh PA Software Engineering Inst.

Marques, R. (2020). A ressignificação da educação e o processo de ensino e aprendizagem no contexto de pandemia da covid-19. Boletim de Conjuntura (BOCA), 3(7):31-46.

Onyema, E., Nwafor, C., Faith, A., Sen, S., Atonye, F., Sharma, A., and Alsayed, A. (2020). Impact of coronavirus pandemic on education. Journal of Education and Practice, 11:108-121.

Riess, M. L. R. (2010). Trabalho em grupo: instrumento mediador de socialização e aprendizagem.

Van Nguyen, D., Pham, G. H., and Nguyen, D. N. (2020). Impact of the covid-19 pandemic on perceptions and behaviors of university students in vietnam. Data in Brief, page 105880 . 